\title{
Evaluación de una propuesta didáctica basada en el trabajo por proyectos para conocer el patrimonio de Cartaya desde la cultura gastronómica: concepciones y perspectivas docentes

\author{
A didactic proposal based on work by projects to know \\ the heritage of Cartaya from gastronomic culture: \\ teaching conceptions and perspectives
}

\author{
Rocío Raposo Camacho, Universidad de Huelva, España (rocioraposo11@gmail.com) \\ Inmaculada Gómez Hurtado Universidad de Huelva, España (inmaculada.gomez@dedu.uhu.es)
}

\begin{abstract}
Resumo: Durante el curso 2019/2020 se realiza un estudio de caso en un centro educativo de la localidad onubense de Cartaya, acogido al proyecto "Los niños se comen el futuro". Se busca evaluar una Propuesta Didáctica, basada en el Trabajo por Proyectos y la integración holística del patrimonio, desde la cultura gastronómica, a partir de las concepciones de docentes de la etapa de Educación Primaria. La muestra la conforma el equipo docente de la etapa y las técnicas de recogida de información son la observación participante, la entrevista, el grupo de discusión y el análisis documental. Los resultados revelan el escaso tratamiento que se le otorga al patrimonio en la educación formal y el buen acogimiento de propuestas metodológicas activas e innovadoras, como la que se presenta, para acercar al alumnado a su entorno, permitiéndole establecer lazos identitarios .
\end{abstract}

Palabras Clave: concepciones docentes, patrimonio, propuesta didáctica, trabajo por proyectos

\begin{abstract}
During the 2019/2020 academic year, a case study is carried out in an educational center in the town of Cartaya, hosted by the project "Children eat the future". It seeks to evaluate a Didactic Proposal, based on Work by Projects and the holistic integration of heritage, from the gastronomic culture, from teaching conceptions of the Primary Education stage. The sample is made up of the teaching team belonging to this stage and the techniques of collecting information are the participant observation, the interview, the focus group and the documentary analysis. The results reveal the limited treatment given to the heritage in the formal education and the good acceptance of active and innovative methodological proposals, such as that presented, to bring to students everything of their environment and allows them to establish identity ties with it .
\end{abstract}

Keywords: heritage, work by projects, didactic proposal, teaching conceptions 


\section{Introducción}

El pasado curso escolar 2018/2019, se desarrolló en los centros escolares de Huelva capital el proyecto educativo "Los niños se comen el futuro", enmarcado en la Fundación PRENAUTA (1), el cual es Pionero Mundial de Educación en Cultura Gastronómica y se encuadra dentro del Programa para la Innovación Educativa, Hábitos de Vida Saludable, Creciendo en Salud de la Consejería de Educación y Deporte de la Junta de Andalucía. El análisis, de este mismo proyecto y de su material, realizado por el grupo de investigación educativa DOCE (2), de la Universidad de Huelva concluye que lo que realmente se desarrolla en la práctica, partiendo de los manuales didácticos, no refleja la filosofía del Trabajo por Proyectos. Por tanto, se presenta una Propuesta Didáctica de intervención educativa como alternativa para este curso, tomando la cultura gastronómica como punto de partida y conformando la perspectiva holística del patrimonio.

Siguiendo a Cuenca et al. (2011), se puede justificar la importancia del acercamiento del patrimonio a través de la metodología del Trabajo por Proyectos, considerándolo como un proceso de aprendizaje significativo que favorecerá que el alumnado construya su identidad cultural, respete, valore y la empatice con otras sociedades, formas de vida y de relación con la naturaleza, permitiendo al docente adaptar el tratamiento de los contenidos al nivel psicoevolutivo de cada niño y cada niña, atendiendo así a la diversidad del aula. Además, con esta propuesta se pretende ampliar los estudios realizados sobre el concepto de patrimonio y su enseñanza en el aula. Esto justifica nuestro objeto de estudio, el cual tiene como fin evaluar una Propuesta Didáctica, basada en el Trabajo por Proyectos y la integración holística del patrimonio, desde la cultura gastronómica, a partir de las concepciones de docentes de la etapa de Educación Primaria, del CEIP Juan Ramón Jiménez de Cartaya.

\section{Marco teórico}

\section{1 ¿Qué se entiende por patrimonio?}

El patrimonio engloba el conjunto de bienes culturales y naturales que heredamos de nuestros antepasa-

\footnotetext{
${ }^{1} \mathrm{https} / /$ fundacionprenauta.org/portfolio-item/los-ninos-se-comen-futuro/ ${ }^{2} \mathrm{http}: / /$ www.uhu.es/doce/
}

dos y que nos permiten entender y conocer nuestra historia, costumbres y formas de vida (UNESCO, 2004). Por eso, se considera la base donde la humanidad construye su memoria colectiva y su identidad, permitiéndole identificarse con una cultura, con una lengua y una forma concreta de vivir que se transmite de generación en generación. Así, la preservación del patrimonio es una necesidad vital para todos los pueblos y tenemos la responsabilidad de protegerlo y transmitirlo, propiciando su disfrute y comprensión.

El concepto de patrimonio ha experimentado una importante evolución desde su origen, que se ha visto reflejada también en el campo educativo. Se habla de muchos tipos de patrimonio, que dependerán de la clasificación que adoptemos. Sin embargo, debemos llegar a una visión integradora, holística del mismo. Para el grupo de investigación en Didáctica de las Ciencias Sociales de la Universidad de Huelva, existe un solo patrimonio que se puede desgranar en estas cinco tipologías: natural, histórico, artístico, científico -tecnológico y etnológico (UNESCO, 2004; Trepat, 2006, citado en Cuenca et al., 2011). Desde nuestra perspectiva de estudio, el patrimonio cultural toma mayor relevancia dada su idoneidad en el marco de la ciudad y de la escuela como escenario educativo (Coma, 2011).

La escuela es la principal institución social en la que confluyen los conocimientos que adquieren los futuros ciudadanos sobre el patrimonio. En este sentido, es importante considerar que la investigación en educación patrimonial ha experimentado un incremento positivo en la última década, prestando atención primordial en su papel como recurso en los procesos de enseñanza-aprendizaje, y articulándose como fuente básica del conocimiento social en ámbitos formales y no formales (Hernández, 2003, citado en Ferreras y Jiménez, 2013).

La educación patrimonial no tiene sentido sin el ciudadano. Siguiendo a Estepa et al., (2005, citado en Lucas y Estepa, 2016), éste es su heredero, el responsable de su transmisión a futuras generaciones, de su defensa y de la mejora del medioambiente, así como un sujeto activo en su disfrute y valoración como señas de identidad. La educación patrimonial conforma un enfoque de carácter multidisciplinar, globalizador, 
integrador y simbiótico, que coloca el acento en la dimensión relacional existente entre los términos "educación" y "patrimonio" y entre los elementos que integran a cada uno de ellos. Todo intento por definir la educación patrimonial y la Didáctica del Patrimonio, debe apoyarse sobre la necesidad de conjugar la interdisciplinariedad. Así mismo, se han de flexibilizar las estrategias didácticas para que se generen procesos de conocimientos, puesta en valor y sensibilización hacia el patrimonio por parte del alumnado. Para ello, se requiere de estrategias necesarias que le permitan conocerlo y comprenderlo (Plan Nacional de Educación y Patrimonio, 2015).

Los movimientos de renovación pedagógica que tuvieron lugar a principios y mediados del siglo XX impulsaron el acercamiento del patrimonio al ámbito educativo, así como su consolidación como instrumento de aprendizaje de las Ciencias Sociales en los diferentes niveles educativos (Coma, 2011). De manera gradual, se ha venido entendiendo y defendiendo el papel activo del tratamiento didáctico del patrimonio, dentro y fuera de las aulas, así como la necesidad de trabajar, de manera coordinada, en instituciones culturales y centros escolares. Además de este interés por potenciar educativamente el patrimonio, lo importante es saber cómo hacerlo y cómo utilizarlo.

\section{2 ¿Cómo educar a través del patrimonio? ¿Qué significa educar mediante el patrimonio?}

Educamos a través y mediante el patrimonio partiendo del contexto en el que nos enmarcamos, de su cultura, sus costumbres y de aquellos aspectos que hacen distintivo a cada lugar, para formar ciudadanos del presente y del futuro más sensibles y más educados; que sepan valorar, cuidar y difundir, desde la infancia, las señas de identidad que configuran el espacio en el que se desenvuelven y, en definitiva, a ellos/as mismos/as como personas. De acuerdo con Cuenca (2016), debemos partir de contenidos que cuenten con una determinada relevancia social para el alumnado. Para ello, la cotidianeidad del patrimonio debe tener un sentido práctico y significativo para éste y se debe incorporar en la escuela a través de talleres, dramatizaciones y el uso de las redes sociales y sus herramientas asocia- das. De esta forma, el alumnado sentirá interés por conocerlo y por intervenir en su defensa y conservación, siendo consciente de la realidad y el contexto en el que está presente este patrimonio. Todo este proceso favorecerá que el alumnado construya su identidad cultural, respete, valore y la empatice con otras sociedades, formas de vida y de relación con la naturaleza (Cuenca et al., 2011).

No obstante, realizando una búsqueda sobre investigaciones anteriores, los resultados obtenidos por Martínez et al. (2019), muestran que queda mucho trabajo pendiente en educación patrimonial para convertir el patrimonio en un recurso provechoso en la enseñanza del alumnado de educación primaria. Teniendo en cuenta estos resultados y, en consonancia con Fontal et al. (2017), es necesaria la formación del profesorado sobre los contenidos patrimoniales para facilitar la transmisión de conocimientos y una mayor sensibilización. En este sentido, siguiendo a Pinto y Molina (2015), los docentes podemos realizar una gran variedad de prácticas a través del contacto directo con el patrimonio local, superando así posibles déficits existentes por parte de la administración educativa, editoriales, docentes y gestores patrimoniales.

\subsection{El aprendizaje del patrimonio a través de la investigación escolar: el Trabajo por Proyectos}

Cuando el interés de la didáctica patrimonial se sitúa en los niños y las niñas, el papel del docente es facilitar el aprendizaje significativo, de manera que los nuevos contenidos se engarcen al conocimiento previo del alumnado. Así, se puede considerar que la metodología del Trabajo por Proyectos se enmarca en uno de esos enfoques que más se aproxima a una concepción constructivista del proceso de enseñanzaaprendizaje del patrimonio. Las características principales de esta metodología son: incorpora al aula escolar abundancia y riqueza de recursos; aprender investigando constituye un revulsivo para repensar la educación y el papel de la escuela; se trata de una tradición innovadora que aborda el currículum partiendo de temáticas relevantes, interesantes y significativas culturalmente para el alumnado; integra conocimientos diversos, mediante un proceso organizado, 
que se muestra abierto a la participación, buscando dar respuestas fundamentadas a cuestiones importantes; es flexible y su evaluación es siempre formativa, apoyándose en el proceso para buscar resultados (Pozuelos, 2007).

En palabras de Cuenca et al. (2017), la educación patrimonial apuesta por la promoción de propuestas didácticas que se apoyen en los principios básicos del modelo investigador, donde sienta sus bases el Trabajo por Proyectos, lo que aúna aún más el énfasis de que este modelo produce buenos resultados y, por tanto, conlleva a la conservación del patrimonio y al hecho de que éste sirva como medio para llegar a conocer, para valorar, disfrutar y usar el patrimonio para nuestra socialización y nuestro desarrollo como ciudadanos críticos.

\section{Marco metodológico}

\subsection{Fundamentación metodológica}

La presente investigación se enmarca en el paradigma interpretativo, pues se interacciona con el entorno y con la situación que se va a estudiar, de manera que el investigador logre interpretar, recrear y reconstruir los significados observados y registrados. Esta cuestión ocasiona que se encuentre dentro de los límites de la metodología de corte cualitativo (Flick, 2018) y se desarrolle mediante un estudio de caso, una herramienta y estrategia metodológica que facilitará y permitirá la observación, el análisis y el estudio exhaustivo del objeto (Stake, 2010): la evaluación de la Propuesta Didáctica diseñada a través de las concepciones sobre patrimonio que tienen los docentes de Educación Primaria y sobre la metodología más idónea que consideran o llevan a cabo para acercar al alumnado al patrimonio local, partiendo del Proyecto de cultura gastronómica que se implementa en el centro en esta etapa educativa. Así mismo, es importante destacar que se trata de una investigación de diseño, pues se ha programado una Propuesta Didáctica que recoge esto mismo, siguiendo la metodología del Trabajo por Proyectos, pero no se ha llevado a la práctica; y un estudio exploratorio porque no existen antecedentes que realicen una crítica de un manual didáctico concreto y que, además, propongan alternativas para trabajarlo de forma significativa e innovadora, a través de una metodología verdaderamente activa.

\subsection{1 ¿En qué consiste el proyecto de cultura gas- tronómica que se está llevando a cabo en el cen- tro?}

La cultura gastronómica emana del denominado patrimonio etnológico que tiene un escaso tratamiento aun tratándose del patrimonio más cercano a la sociedad, vinculado a nuestra forma de vida, nuestra cultura, actividades y modos de producción. Por tanto, desde este proyecto lo que se pretende es acercar al alumnado al patrimonio etnológico de su entorno, a su cultura gastronómica, y de la que se partirá en el diseño de la Propuesta Didáctica para trabajar el patrimonio general de la localidad de Cartaya. De esta forma, se persigue la finalidad principal de educar al alumnado en la construcción de su identidad patrimonial, propiciando el establecimiento de vínculos con aquellos elementos patrimoniales a los que les atribuyan un valor determinado, influidos por sus experiencias y vivencias personales.

\subsection{Objetivos y pregunta de investigación}

Partiendo de la pregunta: ¿es adecuado, bajo el punto de vista de los docentes de Primaria, trabajar la perspectiva holística del patrimonio, partiendo de la cultura gastronómica, a través de una Propuesta Didáctica basada en el Trabajo por Proyectos?, el objetivo general que se persigue es evaluar una Propuesta Didáctica, basada en el Trabajo por Proyectos y la integración holística del patrimonio, desde la cultura gastronómica, a partir de las concepciones de docentes de la etapa de Educación Primaria. En su consecución, se busca alcanzar otros objetivos de carácter específico: (a) estudiar el manual "La ensalada de queso fresco y frutos secos" como una propuesta de inicio al Trabajo por Proyectos para desarrollar el concepto de patrimonio, a través de la cultura gastronómica, en el alumnado de Educación Primaria; (b) diseñar una Propuesta Educativa basada en el Trabajo por Proyectos para trabajar el concepto holístico del patrimonio en el primer curso de Educación Primaria; (c) conocer las concepciones docentes sobre el concepto de patrimonio y su proceso de enseñanza y aprendizaje a través del Trabajo por Proyectos; y (d) contrastar la pertinencia de la Propuesta Didáctica diseñada con las impresiones de los docentes con respecto a la misma. 


\subsection{Fases de la investigación}

Son cuatro las fases fundamentales que van a guiar los pasos sobre los que se va a avanzar en la investigación:

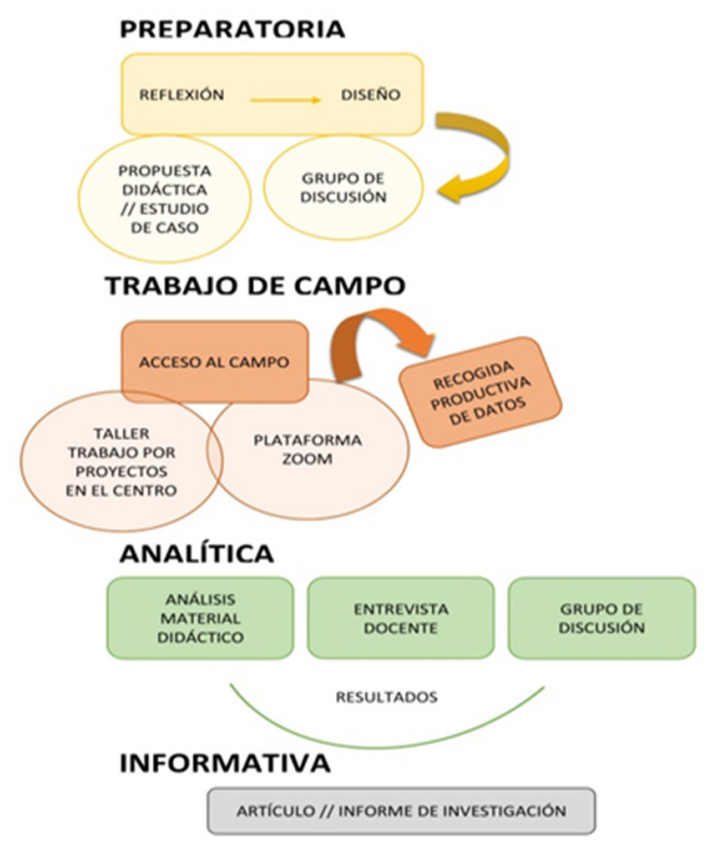

Imagen 1. Fases de la investigación. Fuente: elaboración propia.

1) Fase Preparatoria. Se reflexionó sobre lo que se deseaba investigar y el curso que tomaría la investigación y se analizó el material didáctico. En función de las conclusiones obtenidas de este análisis, se diseñó la Propuesta Didáctica. Esta Propuesta se diseña para su desarrollo durante quince sesiones, a lo largo de un trimestre. Nuestra primera intención es desarrollarla con el primer curso de la etapa, aunque el Taller de Formación se celebra para darla a conocer al resto de maestros/as y así poder hacerla extensible a todo el centro, si la crisis socio-sanitaria acaecida por la Covid-19 no lo hubiera evitado. Así mismo, cabe destacar que el acceso al campo y el tránsito de negociación con los implicados en la investigación se desarrolla gracias al contacto estrecho con la tutora del primer curso; aspecto que facilitó y favoreció la implementación de la investigación con el resto de compañeros del equipo docente, pertenecientes a la etapa de Educación Primaria. 2) Fase de Trabajo de Campo. En un primer momento, se desarrolló un Taller en el centro, organizado y dirigido por las investigado- ras, con los docentes de la etapa, para formarles en las bases fundamentales de la metodología del Trabajo por Proyectos. En un segundo momento, se realizó una videollamada grupal en la plataforma Zoom, para iniciar el grupo de discusión y la entrevista a la tutora del primer curso, destinatarios de la Propuesta Didáctica. Tras la recogida productiva de datos, se llevó a cabo la transcripción de las intervenciones. 3) Fase Analítica. Los datos recabados en el Taller de formación se recogieron en una parrilla de observación previamente elaborada en base al sistema de categorías. Para el análisis de los datos obtenidos en el grupo de discusión y en la entrevista a la docente se establecieron dos colores para catalogar las intervenciones en por categorías. Después, se organizó todo en una tabla. Finalmente, se analizaron y describieron los resultados. 4) Fase Informativa. Se redactó este artículo donde se informa sobre el transcurso y los resultados de la investigación a la comunidad investigativa.

En estas cuatro fases se presentan, de manera muy sistematizada, los pasos que hemos seguido en nuestra investigación. Si bien, podemos acotar, de manera aproximada, el tiempo dedicado a cada tarea: al análisis del material didáctico y su contraste con el análisis previo del mismo se le ha dedicado diez horas, aproximadamente; al diseño de la Propuesta Didáctica y de los instrumentos de observación y registro se le ha dedicado tres meses; la estructura del Taller de Formación fue preparada en una reunión informal de tres horas y la sesión con los docentes duró 90 minutos; por último, la entrevista y la celebración del grupo de discusión ocuparon 120 y 180 minutos del tiempo de investigación, respectivamente. Finalmente, para el análisis, el contraste, la redacción de los resultados y de la discusión se dedicaron dos meses.

\subsection{Contexto}

La fase de trabajo de campo se desarrolla con el equipo docente de la etapa de Educación Primaria del CEIP Juan Ramón Jiménez de Cartaya, una localidad costera situada en la provincia de Huelva. Este centro acoge, aproximadamente, a 750 alumnos/as desde el segundo ciclo de Educación Infantil hasta $6^{\circ}$ de Educación Primaria. La selección de este centro educativo para el desarrollo de la investigación se debe a que, 
entre los proyectos educativos que se llevan a cabo en el centro, se encuentra el Proyecto de Cultura Gastronómica que se pretende estudiar y sobre el que se va a trabajar. Además, otro de los motivos de esta selección hace referencia a la predisposición y el interés por parte de la maestra tutora del grupo para establecer esta filosofía de trabajo no solo en su aula, sino como una propuesta innovadora que se le presenta a todo el profesorado de la etapa de Educación Primaria, con el objetivo de implementar la Propuesta Didáctica diseñada como un verdadero proyecto basado en la filosofía del Trabajo por Proyectos. Atendiendo a la muestra, para el desarrollo de la Propuesta Didáctica que, como apuntamos anteriormente, no se ha llevado a la práctica, se contaría con un grupo de 26 niños y niñas del primer curso de Educación Primaria. Para el tratamiento de la investigación que se realiza finalmente, con el objetivo de evaluar este Diseño de Propuesta Didáctica desde las concepciones de los docentes, se cuenta con el equipo docente de la etapa de Educación Primaria, de los cuales 20 docentes participaron en el Taller de formación, 5 en el grupo de discusión, y la tutora del primer curso de primaria en la entrevista.

\subsection{Instrumentos}

Para el desarrollo de la investigación, se establecen como instrumentos de recogida de datos: (a) un análisis documental del material didáctico y de su informe previo, con un comentario crítico y analítico sobre los datos obtenidos; (b) la observación participante en el desarrollo del Taller de formación sobre Trabajo por Proyectos, impartido a los 20 docentes mencionados anteriormente, para el que se elabora una parrilla de observación; (c) una entrevista estructurada, desarrollada con la tutora, que consta de 13 preguntas; y (d) un grupo de discusión, para el que se proponen un total de 20 cuestiones a debatir con los 5 integrantes del equipo docente.

El instrumento para analizar toda esta información ha consistido principalmente en un sistema de categorías adaptado de EPITEC (Martín y Cuenca, 2015; Cuenca y Estepa, 2017; Arroyo, 2019; Crespo, 2019), que grada de lo simple a lo complejo las subcategorías e indicadores que lo componen. Este sistema de categorías es apto para el análisis de todos los datos recabados, y para evaluar la Propuesta Didáctica. En este sistema se han descrito tres categorías comunes al objeto de estudio de la investigación. La primera categoría (Concepto de Patrimonio) tiene dos subcategorías: la perspectiva del patrimonio y el tipo de patrimonio que se identifica; la segunda categoría (Patrimonio e Identidad) hace referencia a la tipología patrimonial e identidad y a la relación y representación identitaria; en la tercera categoría (Metodología de Enseñanza y Aprendizaje) se presentan subcategorías relacionadas con el papel del docente, el papel del alumnado, los tipos de actividades y los recursos e instrumentos para enseñar y aprender patrimonio. Todos los indicadores de cada subcategoría se establecen en función de diferentes niveles de evolución conceptual en docentes y materiales didácticos, en relación con el patrimonio y su enseñanzaaprendizaje.

\section{Resultados}

Los resultados obtenidos se presentan estructurados en función de las distintas categorías que han guiado la investigación, contrastando en cada categoría y subcategoría la evaluación de la Propuesta Didáctica diseñada a partir de los datos obtenidos mediante los distintos instrumentos de recogida de información. En términos generales, los docentes que han participado en el grupo de discusión y la tutora entrevistada, han evaluado la Propuesta catalogándola positivamente como "muy adecuada y pertinente para trabajar la visión holistica del patrimonio de manera significativa y cercana al nivel psicoevolutivo del alumnado de la etapa", así como "una buena oportunidad para dejar a un lado el libro de texto y dejarse llevar por lo que, tanto al docente como al alumno, les interesa enseñar y aprender respectivamente".

4.1 Categoría I: Concepto de patrimonio. Perspectiva patrimonial y tipos de patrimonio

Tomando como base el análisis realizado sobre el material didáctico para el alumnado, el manual no muestra los contenidos desde una perspectiva patrimonial explícita. No obstante, en cuanto a tipos de patrimonio, se podría considerar que existe una pre- 
sencia del patrimonio etnológico, específicamente de la cultura gastronómica, tratándose así contenidos de las áreas de Ciencias Sociales y Naturales, como la gastronomía, la salud o el conocimiento de los alimentos autóctonos.

Los asistentes al Taller formativo reconocen que no cuentan con una definición clara y exacta sobre el patrimonio. De los 20 asistentes, 5 mostraban una perspectiva patrimonial fetichista-excepcionalista; 5 , monumental; 5, estética; y 5, temporal-histórica. Por tanto, se catalogan dentro de los tipos de patrimonio natural, histórico y artístico, y etnológico.

Analizando los resultados de la entrevista que se le realiza a la tutora, se identifica una perspectiva patrimonial simbólico-identitaria y una visión holística del patrimonio, pues hace referencia a todos los elementos simbólicos que caracterizan a una sociedad, un entorno natural y la geo-biodiversidad, y considera global e integradamente todas las manifestaciones patrimoniales. No obstante, según la consideración de la tutora, los niños y las niñas de $1^{\circ}$ y $5^{\circ}$ de Primaria muestran una perspectiva patrimonial monumental, pues consideran patrimonio solo aquello que "se puede hacer o visitar en el pueblo".

En los resultados del grupo de discusión, la docente 1 y la docente 4 muestran una perspectiva patrimonial simbólico-identitaria, apoyándose ambas en elementos de carácter natural, histórico, artístico y etnológico, en los que se reflejan los tipos de patrimonio que consideran como tal: "pueden ser arqueológicos, artísticos, folclóricos, lingüísticos, gastronómicos...", asegura la docente 1. Según su visión, los niños y las niñas muestran una perspectiva patrimonial estética, en la que establecen fuertes vínculos hacia el patrimonio natural. Por su parte, la docente 4 pretende transmitir una perspectiva patrimonial holística: "este año, al trabajar el concepto de pueblo en la asignatura de Ciencias Sociales, me he centrado en una tarea final que era Vive y Siente Cartaya". La docente 2 y la docente 5 asocian el patrimonio al tiempo, no solo al pasado sino también al presente y al futuro, por lo que muestran una perspectiva patrimonial temporalhistórica. En este sentido, en lo que respecta a la construcción de la perspectiva patrimonial por parte del alumnado, la docente 2 afirma que "necesitamos que el trabajo de conocer el patrimonio esté más organizado". El tipo de patrimonio al que hace referencia es el natural, porque se siente cómoda y le gusta que los niños sean partícipes de ello con juegos al aire libre. Por su parte, la docente 5 hace referencia a la transmisión del patrimonio a través de leyendas o del vocabulario de la localidad. Si bien, a veces alude a elementos cercanos y propios, mostrando una perspectiva fetichista-excepcionalista. La docente 3 no presenta una clara referencia hacia una determinada perspectiva patrimonial. Sin embargo, se pueden catalogar sus afirmaciones como más afines al patrimonio natural, histórico y etnológico, como la docente 5 . Además, menciona los juegos tradicionales de la Pradera de San Isidro o las rumbas, sevillanas y fandangos que les enseña a su grupo de alumnos/as.

Como se puede observar, en relación a la primera categoría, no existe una consonancia entre los resultados recogidos en los distintos instrumentos.

4.2 Categoría II: Patrimonio e identidad. Tipología patrimonial e identidad; relación identitaria; representación identitaria.

Entre los contenidos del material didáctico y el patrimonio se establece una relación identitaria territorial, ya que se presentan fundamentalmente elementos naturales relacionados con la alimentación, que se pueden adquirir del propio medio. No obstante, la representación del patrimonio a través de dibujos o imágenes es aidentitaria, pues carece de algún tipo de relación significativa con el contexto.

Los asistentes al Taller, en su mayoría, se identifican con elementos significativos, tradicionales y de carácter medioambiental. Las relaciones identitarias que establecen con el patrimonio se consideran fundamentalmente afectivo-emocionales, por lo que la representación identitaria está cargada de señas de identidad por vivencias familiares o de la vida cotidiana, y festivas.

No obstante, en relación a los datos analizados en la entrevista a la tutora, atendiendo a la subcategoría de tipología patrimonial e identidad, no se establece clasificación, debido a que afirma que el concepto de patrimonio pasa desapercibido, siendo su presencia anecdótica, tanto en el sistema educativo como en su 
centro concretamente y, por ende, no se trabaja desde la perspectiva que ella defiende. Por el contrario, sí se considera el trabajo que realiza la docente en el aula con su grupo de alumnos/as, y la principal finalidad que persigue es "que conozcan mejor el lugar en el que viven, identificarse y conectarse con sus origenes, y activar y potenciar su sentido de pertenencia para valorarlo, conservarlo, mejorarlo y difundirlo". Así, las relaciones que establece entre el alumnado y el patrimonio se conciben desde la multiplicidad identitaria, dando un paso más allá con respecto al material didáctico y a sus compañeros, aunque conforme una representación del patrimonio como señas de identidad personal. Sin embargo, atendiendo al tratamiento que el patrimonio recibe a nivel general en el centro, la representación del patrimonio es aidentitaria, porque se trabaja de manera puntual a través de efemérides en las que no se transmite el porqué de esa celebración o su importancia en la sociedad. Por lo tanto, explícitamente no se establecen relaciones de identidad.

En el grupo de discusión, la docente 1 se identifica con elementos de carácter medioambiental, significativos y tradicionales, etnológicos y con referentes históricos. A su vez, piensa que el alumnado se identifica más con los elementos naturales de su contexto más cercano. La relación identitaria que establece con el patrimonio es afectivo-emocional, porque lo relaciona constantemente con el pueblo y con las personas que lo habitan. Por tanto, la representación identitaria que establece del patrimonio es a partir de señas de identidad configuradas a través de vivencias familiares. La docente 2 y la docente 3 muestran una identificación con elementos significativos y tradicionales, en este caso, los juegos. Además, la docente 3 menciona elementos de carácter medioambiental, destacando que "a los niños les entusiasma y encanta". La relación identitaria que establecen estas dos docentes con el patrimonio viene marcada principalmente por las relaciones familiares, el sentimiento de pertenencia al mismo pueblo y por los elementos naturales y espaciales que lo componen. Estos aspectos conllevan a una representación identitaria del patrimonio a partir de señas de identidad. La docente 4 y la docente 5 se identifican con el patrimonio histórico y et- nológico. La primera hace referencia a los siguientes elementos patrimoniales: "yo me quedo con el patrimonio etnológico, tradiciones, romerías, fiestas patronales, comidas, bailes...". La segunda se identifica con "la gastronomía, los dialectos del pueblo y las leyendas". La relación identitaria que establecen con el patrimonio está determinada por referentes patrimoniales asociados a las relaciones familiares y sociales. Ambas docentes han desarrollado señas de identidad hacia el patrimonio de su localidad y de la localidad en la que trabajan.

Considerando estos resultados, puede deducirse de manera contradictoria que, a pesar del tratamiento superficial y casi inexistente que se le otorga al patrimonio en el centro a nivel general y a través de los contenidos del manual prediciéndose una representación aidentitaria del mismo, puede comprobarse cómo a la hora de trabajarlo en el aula los docentes muestran un interés especial en acercar el patrimonio a los niños como aquello que forma parte de las costumbres y de la vida de las personas de su contexto y que, por tanto, son señas que les otorgan una determinada identidad fuertemente marcada por las relaciones afectivoemocionales.

\subsection{Categoría III: Metodología de enseñanza/} aprendizaje. Papel del docente; Papel del alumnado; Tipos de actividades; Recursos e instrumentos

El material didáctico está diseñado para trabajar la cultura gastronómica a través de la filosofía del Trabajo por Proyectos. Sin embargo, su organización y su propuesta didáctica es propia de la metodología magistral y convencional. En general, las actividades que proporciona son cerradas e intuitivas, de "lápiz y papel”. No invitan a la duda, a la investigación y a la experimentación del alumnado para que construya su propio aprendizaje, sino que siguen un patrón tradicional; no se le otorga total libertad para expresar lo que ha aprendido ni potencia el trabajo cooperativo y colaborativo.

Los asistentes al Taller se mostraron interesados en la metodología del Trabajo por Proyectos y en la implementación de la Propuesta Didáctica en los distintos niveles de Educación Primaria, pues la consideran una oportunidad de enseñanza-aprendizaje significativa que 
se aleja del convencionalismo que actualmente impera en el centro educativo. Así mismo, reconocían que, a pesar de procurar desarrollar actividades innovadoras y atractivas para el alumnado, no siempre consiguen apartar la metodología tradicional de su día a día.

Los datos obtenidos en la entrevista a la tutora reflejan que ésta considera su papel activo en el aula, dentro de las circunstancias que se lo permitan, de manera que participa como guía, reconductora y motivadora. Por su parte, le concede al alumnado un papel activo en tareas y actividades de investigación, favoreciendo el trabajo en equipo, la toma de decisiones y el desarrollo de su espíritu emprendedor. En cuanto al tipo de actividades, desarrolla tareas y experiencias innovadoras solo puntualmente y en la intimidad de su clase, a nivel particular, y utiliza recursos TIC como la pizarra digital, y otras aplicaciones de Google.

Del grupo de discusión se pueden destacar resultados dispares. La docente 1 reconoce que abandonaría su papel instructor para adoptar un papel más dinámico y activo. En este sentido, subraya el papel pasivo del alumnado: "los niños juegan un papel realmente pasivo en el proceso educativo". Las actividades que se desarrollan son, en consecuencia, actividades de tipo tradicional, aunque a veces se llevan a cabo otro tipo de dinámicas, como salidas, talleres, proyectos puntuales, etc. En cuanto a los recursos e instrumentos, suelen ser materiales tradicionales, como fotos, PowerPoint, imágenes, etc. Sin embargo, también menciona aquellos de uso más innovador, como los propios elementos del medioambiente o cuadernos de registros. Así mismo, enumera varios ejemplos de recursos humanos, propios de las salidas al entorno externo a la escuela, como monitores, asociaciones, etc. La docente 2 afirma que emplea una metodología más tendente a la innovación, estimulando, fundamentalmente, el sentido de iniciativa del alumnado, considerándolo primordial en la enseñanza-aprendizaje del patrimonio: "el papel que el niño puede jugar en un momento dado como ciudadano, es decir, siendo parte del medioambiente, si no les gusta algo, preguntándonos cómo podemos cambiarlo". Teniendo en cuenta estas afirmaciones, docente y alumno juegan un papel activo en el proceso de enseñanza-aprendizaje. Así mismo, los tipos de actividades que menciona son charlas de los abuelos o tareas a través de proyectos. Como materiales y recursos agrega el propio patrimonio local, ya que lo considera un vehículo esencial para el aprendizaje, y también hace mención a mapas conceptuales, un instrumento básico para el aprendizaje mediante el Trabajo por Proyectos. La docente 3 considera fundamental el papel activo del docente en el proceso de enseñanza-aprendizaje del patrimonio pues defiende que, si éste no lo conoce, no podrá enseñarlo. No obstante, reconoce que la realidad es completamente diferente a las expectativas en cuanto a las metodologías activas. Como tipos de actividades que ha llevado a cabo en la intimidad de su aula para enseñar patrimonio, menciona los juegos populares y la visualización de videos de coros de niños cantando fandangos. Como recursos e instrumentos, cita el carácter tradicional otorgado al tratamiento de los contenidos del libro de texto e incluye algunos recursos innovadores que ha implementado de su impronta, como "el aprendizaje de rumbas, sevillanas, juegos fisicos tradicionales, a través de distintas dinámicas activas". La docente 4 defiende su papel activo, motivador y guía en el proceso de enseñanza-aprendizaje y, por tanto, el papel activo, participativo y el protagonismo del alumnado. Como tipos de actividades, menciona aquellas ligadas a la experimentación, como los juegos populares, la tarea de "Vive y siente Cartaya" o las salidas al exterior. Como recursos e instrumentos, reconoce utilizar tanto aquellos que son de uso innovador (programas de radio) como aquellos de uso tradicional (dibujos). La docente 5 reafirma el papel activo que desempeña frente al papel pasivo del alumnado. Sin embargo, defiende que, en la intimidad de su aula, procura otorgarle un papel participativo, experimental y vivencial al alumnado en su propio aprendizaje, favoreciendo así el desarrollo de la autonomía y el sentido de la iniciativa personal. Como tipos de actividades menciona aquellas más innovadoras, como plantar árboles, proyectos de trabajo puntuales, tertulias, etc. Por otro lado, en lo que se refiere a recursos e instrumentos, menciona aquellos de carácter tradicional, como cuadros, imágenes, láminas, fotos, cuadernillos de la historia del pueblo, etc. Reconoce que "hacen falta recursos innovadores $y$ atractivos para trabajar el patrimonio". 
Con los resultados presentados en esta categoría, vemos una coincidencia entre el diseño del manual didáctico sobre cultura gastronómica y las consideraciones de los asistentes al Taller de formación; en ambos casos existe la intención de trabajar bajo la metodología del Trabajo por Proyectos. Sin embargo, esta intención no se desarrolla en la práctica, sino que la metodología convencional aparece "disfrazada" de actividades novedosas y puntuales que no consiguen abrirse paso en la cotidianidad de la actividad lectiva en la escuela. Por su parte, este no es el caso de la tutora entrevistada. Por último, atendiendo a los resultados del grupo de discusión, encontramos resultados dispares, aunque, en general, las docentes que forman parte del mismo reconocen que les gustaría alejar de su aula el convencionalismo que viene imperando en su metodología.

\section{Discusión}

Uno de los principales aspectos que se han detectado durante el transcurso de la investigación, en concordancia con Estepa et al. (2005), hace referencia a que habitualmente en el ámbito educativo se tratan los diversos elementos patrimoniales desde las Ciencias Sociales predominando un enfoque individualista, que no permite el establecimiento de relaciones entre CienciaTecnología-Sociedad-Ambiente (Cuenca et al., 2011). En primer lugar, en este estudio se destaca que no se trabaja el concepto de patrimonio explícitamente en el material didáctico, no aparecen claras referencias a la perspectiva patrimonial ni a los tipos de patrimonio. Por el contrario, en el estudio realizado por Cuenca et al. (2017), se afirma que en los libros de Conocimiento del Medio de Educación Primaria predomina una perspectiva monumental, excepcionalista y, eventualmente, una perspectiva simbólico-identitaria. Sin embargo, la investigación realizada sí coincide con la llevada a cabo por Ferreras y Jiménez (2013), quienes determinan que la visión que se transmite del patrimonio en los libros de texto es muy simple, pues no aparecen elementos vinculados al mismo desde una perspectiva holística y heterogénea, pues la perspectiva patrimonial está marcada por la escasez, grandiosidad y su carácter natural o histórico. Por un lado, en lo que respecta al patrimonio tecnológico, no son demasiado abundantes las prácticas do- centes y los materiales didácticos que utilicen esta tipología, reafirmando el estudio de Cuenca, et al. (2011). Por otro lado, la mayoría de los docentes no cuentan con una definición clara y exacta del patrimonio: algunos se catalogan dentro de una perspectiva fetichistaexcepcionalista, otros tienden más hacia una perspectiva temporal-histórica, y solo en tres casos, se identifica una perspectiva patrimonial simbólico-identitaria. Estos resultados coinciden con las afirmaciones de Cuenca, et al. (2017), ya que son pocos los docentes que podrían ubicarse en el nivel de referencia o perspectiva simbólico-identitaria. Además, su investigación arroja que esto puede deberse a la débil o nula formación de los docentes, como recogemos en nuestros resultados.

En segundo lugar, siguiendo la investigación abordada por Cuenca et al. (2017), en los materiales didácticos los elementos identitarios son comunes, teniendo un determinado valor social y vinculándose al patrimonio etnológico. Por otro lado, se detecta una menor identificación de los docentes con elementos etnológicos, en detrimento de una mayor valoración hacia los naturales y los de carácter histórico-artístico. Por el contrario, en la investigación que se ha realizado, las relaciones que establece el material didáctico con los contenidos son claramente aidentitarias y los docentes manifiestan una identificación, especialmente marcada por vivencias familiares y sociales, con todos los elementos patrimoniales, con menor presencia del artístico y con excepción del patrimonio científico -tecnológico, al que no hacen mención en sus intervenciones. No obstante, cabe destacar que sí se coincide con la investigación de estos autores cuando hacen alusión a la consideración de los docentes sobre su importante responsabilidad.

Por último, la investigación desarrollada por Cuenca et al. (2011) considera la investigación escolar como la metodología más adecuada para propiciar aprendizajes de carácter significativo, funcional y globalizado, donde la exploración y la investigación del entorno constituyen el eje fundamental de la secuencia de actividades desarrolladas en el aula. En este sentido, coincidiendo con la investigación desarrollada por Sanahuja et al. (2015), cada vez son más los docentes que valoran positivamente la implementación de la metodología del Trabajo por Proyectos para enseñar patrimonio en el aula, 
dado que se trata de una metodología que permite que tanto docentes como familiares y expertos participen en las experiencias, favoreciendo el aprendizaje del alumnado. En relación con el papel del docente y del alumnado, en el proceso de enseñanza y aprendizaje del patrimonio se suscriben los resultados de la investigación llevada a cabo por Cuenca et al. (2017): "el protagonismo corresponde al profesorado, mientras que el alumnado actúa como receptor de la información que se transmite verbalmente" (p. 148). No obstante, los docentes de Primaria han defendido que, en la intimidad de su aula, procuran otorgarle un papel activo, dinámico, participativo y protagonista al alumnado. En cuanto a los tipos de actividades, siguiendo a Cuenca et al. (2017), son las salidas de campo y visitas guiadas las actividades más realizadas, a las que añadimos juegos tradicionales o populares, leyendas y diferentes dialectos propios del entorno. Por eso, según Cuenca et al. (2011), no se consideran abundantes las experiencias ni los materiales en los que se desarrollan propuestas de educación patrimonial bajo premisas holísticas, simbólico-identitarias, transdisciplinares y de carácter investigativo. Coincidiendo con esta investigación y la desarrollada por Aranda et al. (2010): "el libro de texto gana seguidores y se erige como el verdadero referente de la reforma, lo que va en detrimento del estudio del Patrimonio más cercano" (p. 10).

\section{Conclusiones}

Comenzando por el material didáctico Cultura Gastronómica I: La ensalada de queso fresco y frutos se$\cos$, se encuentra que su carácter es aséptico, puesto que se trata de un diseño "de laboratorio", convencional, donde se pretende trabajar la cultura gastronómica, pero no se realiza referencia alguna al contexto en que se desarrolla (nivel nacional, autonómico), por lo que se podría trabajar con él en cualquier otro país, tan solo adaptando el idioma. En este sentido, es artificial, por lo que no se encuentra ningún tipo de patrimonio explícito en él. Además, tampoco aparecen vínculos o relaciones con la dieta mediterránea, en la que se incluye el plato de la ensalada, de manera que permita crear una determinada identidad en el alumnado o una asociación explícita con el patrimonio etnológico. Las investigaciones que se han desarrollado en los últimos años ponen de manifiesto cómo los libros de texto monopolizan el trabajo de los contenidos (Ferreras y Estepa, 2012), y el tratamiento que recibe el patrimonio se encuentra sesgado, con un fuerte carácter disciplinar y un tratamiento anecdótico, estableciéndose escasas relaciones identitarias (Cuenca et al. 2011). Tomando en cuenta estas consideraciones, a pesar de que el material pretende acercarse al Trabajo por Proyectos, la metodología tradicional continúa imperando frente al aprendizaje basado las señas de identidad de esta filosofía de trabajo. Por eso, el llegar a implementar el tratamiento del patrimonio en el aula consiguiendo que el alumnado establezca lazos identitarios, dependerá de manera muy significativa de la forma en la que el docente lo presente, la metodología que emplee para tratar los contenidos y cómo se desarrollen las dinámicas en el aula (Gómez et al. 2019). Así, se presenta una propuesta didáctica basada verdaderamente en los principios de la metodología del Trabajo por Proyectos, tomando como base la cultura gastronómica para trabajar el concepto global de patrimonio local de manera integrada y holística. De esta forma, nuestro énfasis en el Taller se concentró en demostrar que se trata de una metodología que requiere interés, esfuerzo y mucho trabajo, pero que favorece significativamente los aprendizajes y la motivación. Demostrar, por tanto, que otro tipo de educación es posible.

En cuanto a las conclusiones obtenidas durante la experiencia en el Taller, la entrevista realizada a la tutora y el grupo de discusión celebrado se destaca que tan solo varios casos se catalogan en una perspectiva patrimonial simbólico-identitaria. No obstante, hacen mención a distintos tipos de patrimonio con los que más se identifican, en la mayoría de los casos por una relación identitaria afectivo- emocional que se han convertido en señas de identidad para ellos con el paso del tiempo. Cabe destacar que el patrimonio natural es el tipo de patrimonio que consideran más importante y significativo. No se hacen referencias al patrimonio científico-tecnológico. En este sentido, resulta interesante cómo consideran patrimonio al dialecto propio del contexto en el que viven. A pesar de todo ello, al no conocer exactamente qué engloba el término de patrimonio, no se trabaja explícitamente 
en el centro a nivel general, otorgándole un tratamiento limitado a la celebración de efemérides, a proyectos de trabajos puntuales o a aprendizajes ocasionales. En relación con la metodología de enseñanzaaprendizaje, muestran un concepto equívoco de la metodología del Trabajo por Proyectos, pues no es lo mismo hablar de trabajo por proyectos que de proyectos de trabajo, siendo éstos últimos los que suelen estar algo más presentes en el aula de manera puntual. Los docentes se acogen en su mayoría al problema ligado a la falta de tiempo para la investigación y la exploración del alumnado sobre los elementos que forman parte de su contexto. En consonancia con los resultados de la investigación de Cuenca et al. (2017), "los docentes de Educación Primaria confirman [...] que en general el profesorado no siente una verdadera preocupación por la didáctica del patrimonio" ( $\mathrm{p}$. 149). Por esta razón, reconocen la necesidad de apoyo $\mathrm{y}$ formación durante todo el proceso para poder desarrollar efectivamente el proyecto, pues les resultaría muy fácil desvincularse de su propósito y volver a las actividades típicas. Teniendo en cuenta estas afirmaciones por parte de los docentes que forman parte de la investigación, sería conveniente observar y conocer el uso que en realidad les otorgan a los recursos cuando defienden que "algunos recursos innovadores sí que se utilizan en el aula"; si invitan a la manipulación y a la experimentación o si finalmente no lo utilizan con eficacia y terminan funcionando como recursos tradicionales e intuitivos. Con todo ello, la evaluación de la Propuesta Didáctica concluye con la idea de que el valor que se le otorga es escueto y superficial debido a la falta de formación con la que cuentan los docentes en relación con el Trabajo por Proyectos. Es decir, a pesar de evaluarla como "muy adecuada y pertinente para trabajar la visión holística del patrimonio de manera significativa", los docentes que forman parte de la investigación no la implementarían en el aula por tres causas principales: la falta de conocimientos sobre el Trabajo por Proyectos; la falta de organización y coordinación; y la falta de una definición clara, exacta y sólida del patrimonio.

Desde nuestro punto de vista y coincidiendo con los docentes, la Propuesta Didáctica que se presenta es adecuada y pertinente para trabajar el patrimonio de forma holística, por razones que se fundamentan y justifican atendiendo a las categorías que han apoyado el transcurso de la investigación. La primera razón está en la presentación explícita del concepto de patrimonio al alumnado en cada una de las actividades que se integran en el Proyecto. Esto puede observarse, por ejemplo, en la actividad de motivación "Escape Room", en la que el alumnado debe expresar la importancia del patrimonio de su localidad, apoyándose en los espacios más importantes de ésta, los platos típicos, los personajes ilustres que forman parte de su historia o las fiestas que se celebran, culminando la actividad con una opinión en la que expongan por qué recomiendan su pueblo para vivir en él. Además, teniendo en cuenta que los docentes que han participado en la investigación no han considerado el patrimonio científico-tecnológico en sus intervenciones, es conveniente destacar la tarea en la que se propone visitar el huerto del familiar de un alumno para observar la técnica innovadora de riego solar "Kondenskompresor". Por tanto, se integra de manera simbólico-identitaria y holística la perspectiva patrimonial y los tipos de patrimonio, respectivamente. Otra de las razones es que se le otorga al alumnado la oportunidad de establecer y descubrir el sentimiento de identidad con el patrimonio de su localidad, pues se establece una multiplicidad identitaria como puede observarse, por ejemplo, en las tareas donde se visita la frutería de un familiar, los bares y restaurantes para conocer recetas típicas del pueblo, o en la que se elabora la ensalada en las inmediaciones del Castillo de los Zúñiga, en la fase de recapitulación y cierre del Proyecto. Esto ocasiona el claro establecimiento de señas de identidad en los niños y niñas. Por último, es una Propuesta válida porque se acoge verdaderamente a las bases de la metodología del Trabajo por Proyectos, como puede atenderse si se analiza la metodología de enseñanza y aprendizaje que sigue la misma. El docente y el alumnado adoptan un papel activo durante todo el proceso, de manera que el aprendizaje es bidireccional. Las actividades son innovadoras y especialmente flexibles, puesto que permiten al docente adaptar el tratamiento de los contenidos a las características psicoevolutivas del alumnado en cada nivel educativo, así como presentarle los contenidos de manera significativa y cer- 
cana. En este sentido, los recursos e instrumentos que se proponen se obtienen directamente del medio, están presentes en su vida cotidiana y se establecen interacciones a diario. En definitiva, resulta interesante cerrar la presente investigación resaltando la importancia de los docentes y su implicación, la existencia de propuestas innovadoras, firmes y sólidas para trabajar el patrimonio, y la colaboración de todos.

\section{Referencias}

Aranda-Campos, A., del Pino-Espejo, M. J. y Montes-Tubío, F. (2010). Los aspectos patrimoniales en la educación primaria en la nueva reforma educativa de Andalucía (España). Revista Iberoamericana de Educación, 52(1), 1-11. https://doi.org/10.35362/rie5211807

Arroyo-Mora, E. (2019). Concepciones y emociones sobre patrimonio en Educación Infantil: análisis de una propuesta educativa. [Trabajo de Fin de Máster no publicado]. Universidad de Huelva.

Coma-Quintana, L. (2011). Actividades educativas y didáctica del patrimonio en las ciudades españolas. Análisis, estado de la cuestión y valoración para una propuesta de modelización. [Tesis Doctoral, Universidad de Barcelona]. https://hdl.handle.net/10803/52205

Crespo-Torres, B. (2019). Conociendo nuestras raíces. Una experimentación educativa en el IES SALTÉS, Punta Umbría. [Trabajo de Fin de Máster no publicado]. Universidad de Huelva.

Cuenca-López, J. M. (2016). Escuela, patrimonio y sociedad. La socialización del patrimonio. UNES: Universidad, Escuela y Sociedad, (1), 22-41. https://hdl.handle.net/10481/58775

Cuenca-López, J. M. y Estepa-Giménez, J. (2017). Educación patrimonial para la inteligencia territorial y emocional de la ciudadanía. Midas, 8. https://doi.org/10.4000/midas.1173

Cuenca-López, J. M., Estepa-Giménez, J. y Martín-Cáceres, M. J. (2011). El patrimonio cultural en la educación reglada. Patrimonio Cultural de España, (5), 45-88. https://hdl.handle.net/10272/9437

Cuenca-López, J. M., Estepa-Giménez, J. y Martín-Cáceres, M. J. (2017). Patrimonio, educación, identidad y ciudadanía. Profesorado y libros de texto en la enseñanza obligatoria. Revista de Educación, (375), 136159. https://hdl.handle.net/10272/13629

Estepa-Giménez, J., Wamba-Aguado, A. M. y Jiménez-Pérez, R. (2005). Fundamentos para una enseñanza y difusión del patrimonio desde una perspectiva integradora de las ciencias sociales y experimentales. Investigación en la Escuela, (56), 19-26. https://hdl.handle.net/10272/10694

Ferreras-Listán, M. y Estepa-Giménez, J. (2012). El Patrimonio en los libros de texto y en el currículum oficial de conocimiento del medio natural, social y cultural. El caso de Andalucía. En O. Fontal-Merillas, P. Ballesteros y M. Domingos (Eds.). I Congreso Internacional de Educación Patrimonial Mirando a Europa: estado de la cuestión y perspectivas de futuro. Madrid: Instituto del Patrimonio Cultural de España (pp. 555-563). https://doi.org/10.13140/rg.2.1.3148.2720 
Ferreras-Listán, M. y Jiménez-Pérez, R. (2013). ¿Cómo se conceptualiza el patrimonio en los libros de texto de Educación Primaria? Revista de Educación, (361), 591-618. https://doi.org/10.4438/1988-592x-re-2013$361-234$

Flick, U. (2018). Designing Qualitative Research ( ${ }^{\text {nd }}$ Ed.). SAGE.

Fontal-Merillas, O., Ibáñez-Etxeberria, A., Martínez-Rodríguez, M. y Rivero-Gracia, P. (2017). El patrimonio como contenido en la etapa de Primaria: del currículum a la formación de maestros. Revista Electrónica Interuniversitaria de Formación del Profesorado, 20(2), 79-95. https://doi.org/10.6018/reifop/20.2.286321

Gómez-Hurtado, I., González-Falcón, I. y García-Rodríguez, P. (2019). Análisis de material didáctico: Cultura gastronómica, el gazpacho andaluz. [Trabajo no publicado]. Grupo de Investigación Educativa DOCE. Universidad de Huelva.

Jiménez, C. (2019). Cultura Gastronómica 1: la ensalada de queso fresco y frutos secos. Madrid: Anaya.

Martín-Cáceres, M. J. y Cuenca-López, J. M. (2015). Educomunicación del patrimonio. Educatio Siglo XXI, 33 (1), 33-54. https://doi.org/10.6018/j/222491

Martínez, M., Salmerón, J. y Serrano, M. (2019). Estudio sobre el patrimonio de la ciudad de Lorca en educación primaria. Revista de humanidades, (38), 11-36. https://doi.org/10.5944/rdh.38.2019.20430

Lucas-Palacios, L. y Estepa-Giménez, J. (2016). El patrimonio como instrumento para la formación de la ciudadanía crítica y participativa. Investigación en la Escuela, (89), 35-48. https://doi.org/10.12795/ ie.2018.189.03

Pinto, H. y Molina-Puche, S. (2015). La educación patrimonial en los currículos de ciencias sociales en España y Portugal. Educatio Siglo XXI, 33(1), 103-128. https://doi.org/10.6018/j/222521

Plan Nacional de Educación y Patrimonio (2015). Ministerio de Educación, Cultura y Deporte. www.mecd.gob.es

Pozuelos, F. J. (2007). Trabajo por proyectos en el aula: Descripción, investigación y experiencias (1a. ed.). Morón de la Frontera (Sevilla): M.C.E.P.

Sanahuja-Ribés, A., Peiro-Rodríguez, M. y Piquer-Gumbau, A. (2015). La prehistoria: una experiencia de trabajo por proyectos en educación primaria. Quaderns digitals, (81), 6-23. https://hdl.handle.net/10234/147825

Stake, R. E. (2010). Qualitative research: Studying how things work. New York: Guilford Press.

UNESCO (2004). La UNESCO y el Patrimonio Mundial. Paris: Ediciones Unesco. 